\title{
Negative Curvature and Exotic Topology
}

\author{
F.T. Farrell, L.E. Jones, and P. Ontaneda
}

\section{Introduction}

We begin with a very basic and natural question, expressed in purposely vague language:

(1.1) Existence: When does a space admit a geometric structure that satisfies a given property $P$ ?

This is usually a very difficult problem. If we do not know the answer (and, in some cases, even if we do) we can continue to study this problem after making additional assumptions. We mention here two ways:

\section{Rigidity and Flexibility.}

If (1.1) is too difficult to solve it is natural to ask the following:

(1.2) Flexibility: Suppose that space $Y$ looks like space $X$, and $X$ admits a geometric structure that satisfies $P$. Does $Y$ also admit a geometric structure that satisfies $P$ ?

If the answer is affirmative then the property $P$ is "flexible" for $X$ and it propagates to all spaces that look like $X$. On the opposite side we have the following question:

(1.3) Rigidity: Suppose that space $X$ looks like space $Y$ and both admit geometric structures that satisfy $P$. Are $X$ and $Y$ equivalent?

If the answer to this question is affirmative, then property $P$ is "rigid" for $X$, that is, $P$ is satisfied by essentially a unique space, the space $X$, among all that look like $X$.

Of course all these questions depend on what we mean by space, geometric structure, property $P$, looks like, equivalent. For example, common choices for space are: manifold, topological space, simplicial complex, group,...etc. Or

The three authors were partially supported by NSF grants. 
looks like could mean: homotopy equivalent to, homeomorphic to, $P L$-homeomorphic to, .... etc. Also equivalent could mean "equivalent" in the DIF F, PL or TOP categories, that is: diffeomorphic to, $P L$-homeomorphic to, homeomorphic to.

If space $Y$ looks like space $X$ but it is not equivalent to it, then $Y$ is sometimes called an exotic $X$ or a fake $X$. Hence, for instance, if homotopy equivalence implies diffeomorphism for a smooth manifold $X$ then there are no smoothly exotic versions of $X$, and $X$ is smoothly rigid.

Note that the answers to questions (1.2) and (1.3) could be any combination of yes and no. Also, if we consider whether there exist exotic versions of a space, then we get more possibilities. Here are some examples:

1. If property $P$ is not Rigid for the space $X$, then there exists an exotic $X$.

2. If property $P$ is Rigid for $X$ and there exists an exotic $X$, then $P$ is not Flexible for $X$.

3. If property $P$ is Rigid and Flexible for $X$, then there are no exotic versions of $X$. This would give a geometric proof that looks like implies equivalence in this case. (Examples: homotopy equivalence implies homeomorphism, PL-homeomorphism implies diffeomorphism... etc.)

We can also state a stronger version of Question (1.3), by requiring that one of the spaces satisfies only a weaker version $P^{\prime}$ of property $P$ :

(1.4) Strong(er) Rigidity: Suppose that space $X$ looks like space $Y$, that $X$ admits a geometric structure that satisfies $P$ and that $Y$ admits a geometric structure that satisfies the weaker property $P^{\prime}$. Are $X$ and $Y$ equivalent?

The strongest possible Rigidity would happen then when $P^{\prime}$ is trivial, that is, just one of the spaces is assumed to admit a geometric structure that satisfy $P$.

We can make variations of the questions above by quantifying the space $X$. For instance we can ask whether a question as above is true for $X$ satisfying certain property (e.g. $X$ is simply connected, is aspherical, has finite fundamental group,... etc) or simply for any $X$. In this case the question becomes just a question about the geometric property $P$, no particular spaces involved.

\section{Classification.}

Suppose we do know that a certain space $X$ admits a geometric structure that satisfies a given property $P$, that is, the answer to the Existence Question (1.1) is affirmative for $X$ and $P$. Then it is natural to ask:

(1.5) How many geometric structures on $X$ satisfy $P$ ? 
There may be infinitely many geometric structures on $X$ that satisfy $P$, so it is better to propose the following:

(1.6) Classification: Study the space of all geometric structures on $X$ that satisfy $P$.

In this paper we study some of the questions above when property $P$ means: negative sectional curvatures. To be more precise we will ask some of the questions above with the following specifications:

- space: Smooth manifold with empty boundary (mostly closed) and large dimensions;

- geometric structure: complete Riemannian metric;

- property P: negative sectional curvatures (we also consider briefly weaker, stronger or related properties);

- looks like: homotopy equivalent to, homeomorphic to;

- equivalent: homeomorphic, $P L$-homeomorphic, diffeomorphic.

Our purpose here is to survey some results concerning some of the questions above (mainly Rigidity and Classification) with these specifications. It is important to note that the proofs of all these results have as a common denominator the existence of exotic elements in Topology, e.g., exotic differentiable or PL structures, non-vanishing of some of the homotopy groups of the space of stable pseudo-isotopies of the circle...etc.

Acknowledgment. The authors wish to thank Pat Eberlein for his helpful comments on an earlier version of this paper.

\section{Negative curvature, homotopy equivalence and homeomorphism}

Recall that we are making the following specifications in the questions above: looks like will mean homotopy equivalent to or homeomorphic to. Of course homeomorphism implies homotopy equivalence and the converse is, in general, not true. But for closed negatively curved manifolds (dimensions $\neq 3,4)$ F.T. Farrell and L.E. Jones $[\mathbf{1 7}]$ proved that these two conditions are really equivalent. In fact they proved that this is true when just one of the manifolds is nonpositively curved.

THEOREM 1. Let $X$ be a closed nonpositively curved manifold with $\operatorname{dim} \neq$ 3, 4. If $Y$ is closed and homotopy equivalent to $X$ then $Y$ is homeomorphic to $X$.

(Actually Farrell and Jones prove more: if $f: X \rightarrow Y$ is a given homotopy equivalence then it is homotopic to a homeomorphism.)

This is the Strongest Rigidity possible, topologically, for the property: nonpositive curvature (see Question (1.4)). Therefore this result proves Borel's Conjecture for closed nonpositively curved manifolds $(\operatorname{dim} \neq 3,4)$. 
Recall that Borel's Conjecture states that two homotopy equivalent closed aspherical manifolds are homeomorphic.

Hence, in our context (i.e., for negative curvature, nonpositve curvature), there will be no difference in saying homotopy equivalent or homeomorphic. Note that, since Hadamard Theorem implies that nonpositively curved manifolds are aspherical, and aspherical spaces are determined by their fundamental groups, we have the following important fact:

- Let $X$ and $Y$ be complete nonpositively curved manifolds. Then $X$ and $Y$ are homotopy equivalent if and only if they have isomorphic fundamental groups.

Hence we also have that throughout this paper the phrase $X$ and $Y$ are homotopy equivalent can be replaced by $X$ and $Y$ have isomorphic fundamental groups.

\section{Flexibility}

We mentioned before that we will review results concerning mainly the Rigidity and the Classification Questions. The Flexibility Question for negative curvature will be discussed very briefly, and we also mention some results on the flexibility of certain properties related to negative curvature.

First we consider the Flexibility for closed negatively curved manifolds. Well, this is an open problem, so we present it as an important question in this area:

- Flexibility for negative curvature: Suppose that $X$ and $Y$ are homotopy equivalent closed smooth manifolds and $X$ admits a negatively curved Riemannian metric. Does $Y$ admit a negatively curved Riemannian metric?

We do know that finite volume pinched negative curvature is not flexible for non-compact finite volume Riemannian manifolds, but before stating this result let's mention a related example.

- Flexibility for flat Riemannian manifolds, i.e., for zero curvature: Suppose that $X$ and $Y$ are homotopy equivalent closed smooth manifolds and $X$ admits a flat Riemannian metric. Does $Y$ admit a flat Riemannian metric?

The answer to this question is negative (see item 2. after (1.3)), because: (1) Browder [11] showed there exist smoothly exotic tori, and (2) zero curvature is a smoothly rigid property, that is:

THEOREM 2. If $X$ and $Y$ are homotopy equivalent closed Riemannian flat manifolds, then $X$ and $Y$ are diffeomorphic.

This result follows from Bieberbach [8] classification results. Actually from the classical Torus Theorem of Lawson and Yau [40] (or more precisely 
from Yau's Thesis [62]) and Gromoll and Wolf [31], we get a stronger smooth rigidity:

THEOREM 3. If $X$ and $Y$ are homotopy equivalent closed manifolds, $X$ is flat and $Y$ is nonpositively curved then $X$ and $Y$ are diffeomorphic.

That is, we do not need both spaces to be flat, we just need one flat and the other nonpositively curved.

REMARKS.

1. The proof of the Torus Theorem (at least the one given by Lawson and Yau) has such a synthetic flavor that it can be easily generalized to the more general setting of geodesic spaces. Here is the statement: If $X$ and $Y$ are homotopy equivalent, $X$ is a closed flat Riemannian manifold and $Y$ is a compact geodesically complete nonpositively curved space (in the sense of Alexandrov), then $X$ and $Y$ are homeomorphic. Here $Y$ is geodesically complete if every geodesic segment can be extended to a geodesic ray.

2. A weaker property than flatness is admitting an infranil structure. Lee and Raymond [41] proved the smooth rigidity for infranilmanifolds: homotopy equivalent infranilmanifolds are diffeomorphic. Also, a weaker property than that of admitting an infranil structure is admitting an infrasolv structure. Farrell and Jones [18] proved the smooth rigidity for infrasolvmanifolds: homotopy equivalent infrasolvmanifolds, $\operatorname{dim} \neq 4$, are diffeomorphic. And Wilking [60] improved this result by showing the condition "dim $\neq 4$ " can be dropped.

Let's go back to negative curvature. Since a finite volume pinched negatively curved metric on a manifold induce a infranil structure on the cross section of the cusps, we have that the smooth Rigidity of infranilmanifolds mentioned above in item 2 can be used to prove the following result of Farrell and Jones $[\mathbf{1 9}]$ :

THEOREM 4. Let $n>5$ such that $\Theta_{n-1}$ is non trivial. Then there exists a connected smooth manifold $N^{n}$ such that

(i) $N$ is homeomorphic to a complete, noncompact, finite volume real hyperbolic manifold.

(ii) $N$ does not admit a finite volume complete pinched negatively curved Riemannian metric.

REMARKS.

1. Here $\Theta_{m}$ denotes the group of homotopy $m$-spheres. Note that $\Theta_{n-1}$ is non-zero for instance when $n>4$ is even and not of the form $2^{k}-2$. For example, $n$ could be equal to 8 .

2. Recall that a Riemannian manifold has pinched negative curvature if there exist $a<b<0$ such that all sectional curvatures lie in the interval $[a, b]$. 
Therefore finite volume pinched negative curvature is not a flexible geometric property for non-compact manifolds. Note this process can not be used for closed hyperbolic (or negatively curved) manifolds because they do not have cusps.

There are a couple of canonical constructions of closed nonpositively curved manifolds from non-compact finite volume hyperbolic manifolds: (1) the double of a hyperbolic manifold and (2) the ones obtained by cusp closing due to Schroeder [58]. The nonpositively curved manifolds obtained in these ways have negative curvature everywhere but in a hypersurface of codimesion 1 or 2 , respectively. The lack of flexibility of flatness was also used in this context by Aravinda and Farrell [3] (for cases (1) and (2), but only in dim $\geq$ 8 and not in every dimension) and Ontaneda [49] (for the double, dim $\geq 6$ ) to show non-flexibility for nonpositve curvature in these cases:

THEOREM 5. There exist closed nonpositively curved manifolds, $\operatorname{dim} \geq 6$, constructed by doubling that have an exotic structure that does not admit a nonpositively curved Riemannian metric.

A similar Theorem holds replacing doubling by cusp closing.

In Ontaneda [49], examples are given of doubles with three not $P L$ equivalent smooth structures, two of which are nonpositively curved and the third one does not admit a nonpositively curved Riemannian metric. Hence, for these examples nonpositive curvature is neither $P L$-rigid nor flexible.

Before we finish this section we mention a couple of results for a less canonical concept of looks like and for the property nonpositive curvature. Recall that two $P L$ structures on a space that agree on each stratum may not be $P L$-equivalent, because the $P L$-structure also depends on how the $P L$ manifold strata are glued. In fact Anderson and Hsiang [2] found obstructions for these gluings that lie in the lower $K$-Theory of the links of these strata. On the other side, Farrell and Jones [17] proved that the lower $K$-Theory for closed nonpositively curved manifolds (dim $\neq 3,4)$ vanish. This motivates us to state the following Rigidity Question: Let $X$ and $Y$ be two nonpositively curved piecewise flat PL-spaces, and suppose that they are homeomorphic by a homeomorphism that is a PL-equivalence when restricted to each manifold strata. Are $X$ and $Y$ PL-equivalent? In [50] Ontaneda showed that the answer is negative. He also showed that the Flexibility Question has also a negative answer. Hence, for a space to have nonpositive curvature it is also important to know how the strata are glued.

\section{Rigidity}

First let's consider a geometric property that is stronger than that of negative curvature: constant negative curvature. If a Riemannian manifold has constant negative curvature, after rescaling, we can assume that this constant is -1 , thus the manifold is hyperbolic. The answer to the Rigidity 
Question (1.3) for closed hyperbolic manifolds is given by one of the fundamental results in geometry: Mostow's Rigidity Theorem [46], which says that this property is metrically rigid (hence smoothly rigid):

TheOREM 6 (Mostow's Rigidity). Let $f: X \rightarrow Y$ be a homotopy equivalence between closed hyperbolic manifolds of dimension $>2$. Then $f$ is homotopic to an isometry.

So the answer to the (smooth) Rigidity Question (1.3) for constant negative curvature is affirmative. It is natural to ask then, as in Question (1.4), if there is a stronger version of this Rigidity. An obvious choice in (1.4) for the weaker property $P^{\prime}$ is negative curvature (i.e, not necessarily constant). We get the following question:

(1.7) A Stronger Rigidity for hyperbolic manifolds: Suppose that $X$ and $Y$ are homotopy equivalent closed Riemannian manifolds. Assume that $X$ is hyperbolic and $Y$ is negatively curved. Are $X$ and $Y$ diffeomorphic?

Or more generally:

(1.8) Smooth Rigidity for negative curvature: Suppose that $X$ and $Y$ are homotopy equivalent closed negatively curved Riemannian manifolds. Are $X$ and $Y$ diffeomorphic?

Of course an affirmative answer for (1.8) implies an affirmative answer for (1.7). There are some very successful tools from Geometric Analysis that were very promising to prove that (1.8) had an affirmative answer. The most important was the Harmonic Map technique.

A smooth map $k: X \rightarrow Y$ between Riemannian manifolds is harmonic if it is a critical point of the energy functional $\mathcal{E}(k)=\int_{X} \frac{1}{2}|D k|^{2}$. An equivalent definition is that the tension field $\tau_{k}$ of $k$ vanishes everywhere. (The tension field $\tau_{k}$ is a section of the bundle $k^{*} T Y$ and can be defined in the following way: for $x \in X$ choose an orthonormal basis $\left\{v_{i}\right\}$ of $T_{x} X$ and define $\tau_{k}(x)=$ $\sum w_{i}$ were $w_{i}$ is the acceleration vector, at $t=0$, of $k\left(\gamma_{i}\right)$, and $\gamma_{i}$ is the geodesic with $\gamma_{i}(0)=x$ and $\frac{d}{d t} \gamma_{i}(0)=u_{i}$.) Given a map $f: X \rightarrow Y$ between Riemannian manifolds, we can try to associate to it a harmonic map that is the limit $k=\lim _{t \rightarrow \infty} k_{t}$, where $k_{t}$ is the unique solution of the heat flow equation, that is, the PDE initial value problem $\frac{\partial k_{t}}{\partial t}=\tau\left(k_{t}\right), k_{0}=f$. If this limit $k$ exists then it is homotopic to $f$ (the homotopy is $t \mapsto k_{t}$ ).

Now, it follows from the classical result of Eells and Sampson [15] that if $f: X \rightarrow Y$ is a smooth homotopy equivalence between closed negatively curved manifolds the heat flow equation beginning at $f$ converges to a well defined harmonic map $k=\lim _{t \rightarrow \infty} k_{t}$. Moreover, from the results of Hartman [36] and Al'ber [1] it follows that $f$ is homotopic to a unique harmonic map. Therefore the homotopy equivalences in Questions (1.7) and (1.8) 
are homotopic to unique harmonic maps. If both manifolds were hyperbolic then this harmonic map is in fact the isometry of Mostow Rigidity Theorem. Therefore we can use the Harmonic Map Technique to try to prove Mostow's Fundamental result. Indeed the Theory of Harmonic Maps had been very successful in showing rigidity results, see for instance Siu [54], Sampson [53], Hernández [37], Corlette [13], Gromov and Schoen [33], Jost and Yau [39], and Mok, Sui and Yeung [45]. Because of this evidence it seems reasonable that Lawson and Yau conjectured that the answer to (1.8) was affirmative.

But the answer to (1.7) is negative (and thus the answer to (1.8) is also negative). This was proved by counterexamples constructed by Farrell and Jones $[\mathbf{1 6}]$. They proved the following.

THEOREM 7. Let $X^{n}$ be a closed hyperbolic $n$-manifold, $\Sigma$ an exotic $n$-sphere, $n \geq 5$, and $\epsilon>0$. Then there is a finite cover $Z$ of $X$ such that

(1) The connected sum $Z \# \Sigma$ is not diffeomorphic to $Z$.

(2) $Z \# \Sigma$ admits a Riemannian metric with sectional curvatures in $(-1-\epsilon,-1+\epsilon)$.

Note that Theorem 7 above shows that the answer to a strengthened version of (1.7) is also negative: the property $P^{\prime}$ (which, in (1.7) is negative curvature) can be replaced by $\epsilon$-pinched negative curvature ( $\epsilon$ depending only on the dimension).

Since $\Theta_{n}$ is trivial in dimensions $<7$, Theorem 7 does not give counterexamples in dimensions $<7$. Furthermore in these dimensions manifolds are diffeomorphic if and only if they are $P L$-equivalent (with the $P L$ structure induced by the given smooth structure). Hence for dimensions $<7$ the Smooth Rigidity Question (1.8) is equivalent to the following $P L$ version:

(1.9) PL Rigidity for negative curvature: Suppose that $X$ and $Y$ are homotopy equivalent closed negatively curved Riemannian manifolds. Are $X$ and Y PL-homeomorphic?

For a general dimension $n$ a negative answer to (1.9) implies a negative answer for (1.8), because diffeomorphic manifolds are $P L$ homeomorphic. The converse is not true in general, but, as mentioned before, it is true for dimensions $<7$. For example an (smoothly) exotic sphere $\Sigma$ is not diffeomorphic to the corresponding sphere (by definition) but it is $P L$ homeomorphic to it, provided $\operatorname{dim} \Sigma \neq 4$. In fact there are no $P L$-exotic spheres in any dimension $\neq 4$. It follows that $Z$ is $P L$-homeomorphic to $Z \# \Sigma$ for any manifold $Z$, and exotic sphere $\Sigma, \operatorname{dim} \Sigma \neq 4$. Therefore Theorem 7 does not answer Question (1.9). Note that, since diffeomorphism implies PL-homeomorphism and this in turn implies homeomorphism, we have that Question (1.9) lies between the Topological Rigidity for negative curvature (which is true, by Theorem 1) and the Smooth Rigidity (which is false, by Theorem 7). 
The answer to Question (1.9) was proved to be negative by Ontaneda [48] in dimension 6 and later by Farrell, Jones and Ontaneda [22] for every dimension $>5$. Here is the result:

Theorem 8. For every $n>5$ and $\epsilon>0$ there are closed Riemannian $n$-manifolds $X$ and $Y$ such that:

(1) $X$ is homeomorphic to $Y$.

(2) $X$ is not PL-homeomorphic to $Y$.

(3) $X$ is hyperbolic.

(4) $Y$ has sectional curvatures in $(-1-\epsilon,-1+\epsilon)$.

The counterexamples constructed in the proof of Theorem 8 use the results of Millson and Raghunathan [44], based on previous work of Millson $[43]$.

Theorems 7 and 8 were the first in a sequence of results that shed some light on the relationship between the analysis, geometry and topology of negatively curved manifolds. These results showed certain limitations of well-known powerful analytic tools in geometry, such as the Harmonic Map technique, the Ricci flow technique, the Elliptic deformation technique as well as Besson-Courtois-Gallot's Natural Map technique [10]. Here we shall just present briefly the main conclusions of this research. A more complete exposition on this area and how it evolved in time can be found in the survey article [26]. The main results are described in the following 5 items:

1. Recall that the Topological Rigidity for negative curvature is true, by Theorem 1. One can ask whether there is a Harmonic Map proof of this fact, that is, whether Lawson-Yau conjecture is true "topologically":

- Question: Let $f: X \rightarrow Y$ be a homotopy equivalence between closed negatively curved manifolds and let $k: X \rightarrow Y$ be the unique harmonic map homotopic to $f$. Is $k$ a homeomorphism?

It follows from Theorem 8 and the $C^{\infty}$ - Hauptvermutung of Scharlemann and Siebenmann [55], that this unique harmonic map $k$ is not, in general, a homeomorphism. Hence, even though we do know that $f$ is homotopic to a homeomorphism (by Theorem 1), we cannot, in general, obtain a homeomorphism using the Harmonic Map technique, at least not directly.

2. Consider Problem 111 of the list compiled by S.-T. Yau in [61] (it is also Grand Challenge Problem 3.6. in [59]) Here it is a restatement of this problem:

- Problem 111 of [61]. Let $f: X \rightarrow Y$ be a diffeomorphism between closed negatively curved manifolds and let $k: X \rightarrow Y$ be the unique harmonic map homotopic to $f$. Is $k$ a homeomorphism?

Note that the difference between this and the previous question in 1 is that we begin now with a diffeomorphism $f$. Hence in this case the heat flow begins already with a diffeomorphism and we want to know is the limit harmonic map $k=\lim _{t \rightarrow \infty} k_{t}$ is a homeomorphism. The examples 
mentioned in 1 above (given by Theorem 8) are not useful, at least directly, to answer this question because $X$ and $Y$ are not $P L$ equivalent in Theorem 8, hence there is no diffeomorphism between them. The answer to the problem above was proved to be yes when $\operatorname{dim} X=2$ by Schoen-Yau [57] and Sampson [52]. But it was proved by Farrell, Ontaneda and Raghunathan [24] that the answer to this question is, in general, negative. In fact because of Scharlemann's generalization [56] of [55] together with the recent positive solution of the Poincaré Conjecture the maps $k_{t}$ are also all nonunivalent for $t$ sufficiently large.

3. Since a harmonic map (between closed negatively curved manifolds) homotopic to a diffeomorphism is not necessarily a homeomorphism we can ask a deeper question: suppose now that the harmonic map can be approximated by homeomorphisms (or even diffeomorphisms), that is, the harmonic map is cellular. Does this imply that the harmonic map is a diffeomorphism? Farrell and Ontaneda [25] showed that the answer to this question is also negative.

4. It was pointed out by M. Varisco that most of the results mentioned above for harmonic maps can also be applied to the natural maps defined by G. Besson, G. Courtois and S. Gallot [10].

5. In items 1-4 above we dealt with processes that produce some special type of map, e.g harmonic maps or natural maps. We can also consider some processes that produce a special type of metric: Einstein metrics, that is, metrics of constant Ricci curvature. The best known method for obtaining Einstein metrics is the Ricci flow method introduced by Hamilton in his seminal paper [34]. Starting with an arbitrary smooth Riemannian metric $h$ on a closed smooth $n$-dimensional manifold $X^{n}$, he considered the evolution equation $\frac{\partial}{\partial t} h=\frac{2}{n} r h-R i c$, where $r=\int R d \mu / \int d \mu$ is the average scalar curvature ( $R$ is the scalar curvature) and Ric is the Ricci curvature tensor of $h$. For $n=3$ Hamilton proved that if the initial Riemannian metric on $X^{3}$ has strictly positive Ricci curvature it evolves through time to a positively curved Einstein metric $h_{\infty}$. This implies that $X^{3}$ equipped with $h_{\infty}$ is a spherical space-form; i.e., its universal cover is the round sphere. Following Hamilton's approach G. Huisken [38], C. Margerin [42] and S. Nishikawa [47], proved that Riemannian manifolds whose sectional curvatures are pinched close to +1 (the pinching constant depending only on the dimension) can be deformed, through the Ricci flow, to a spherical-space form. Then it was natural to ask whether the same was true for Riemannian manifolds whose sectional curvatures are pinched close to -1 , again the pinching constant depending only on the dimension:

- Question: Is there a constant $\epsilon_{n}$, depending only on $n$, such that if $g$ is a Riemannian metric on a closed $n$-manifold with sectional curvatures in $(-1-\epsilon,-1+\epsilon)$, with $\epsilon<\epsilon_{n}$, then the Ricci flow beginning at $g$ converges to an Einstein metric of negative sectional curvatures? 
Rugang Ye [64] proved that sufficiently pinched to -1 manifolds can be deformed, through the Ricci flow, to hyperbolic manifolds, but the pinching constant in his Theorem depends on other quantities (e.g. the diameter or the volume). It was shown later by Farrell and Ontaneda [27], using the tools developed in [25], that the pinching constant cannot depend solely on the dimension by giving examples of arbitrarily pinched to -1 Riemannian metrics for which the Ricci flow does not converge smoothly to a negatively curved metric:

Theorem 9. Given $n>10$ and $\epsilon>0$ there is a closed smooth $n$ dimensional manifold $X$ such that

(i) $X$ admits a hyperbolic metric.

(ii) $X$ admits a Riemannian metric $h$ with sectional curvatures in $[-1-\epsilon,-1+\epsilon]$ for which the Ricci flow does not converge smoothly to a negatively curved Einstein metric.

As mentioned earlier, a more complete account of the results mentioned in the previous five items can be found in $[\mathbf{2 6}]$.

We now give two different versions of Theorem 7: one version for noncompact finite volume complete hyperbolic manifolds and the other for negatively curved manifolds not homotopy equivalent to a closed locally symmetric space. Let us begin with the former. First note that taking the connected sum of a noncompact manifold $X$ with an exotic sphere can never change the differential structure of $X^{n}, n>4$. Therefore we do not have an exact analogue of Theorem 7 for the finite volume noncompact case. Still, Farrell and Jones [20] considered Dehn surgery along a properly embedded tube $\mathbb{S}^{1} \times \mathbb{D}^{n-1}$ in $M^{n}$ to prove:

THEOREM 10. For every integer $n$ such that $\Theta_{n-1} \neq 0$ and any $\epsilon>0$ there are non-compact Riemannian $n$-manifolds $X$ and $Y$ with finite volume such that

(i) $X$ is homeomorphic to $Y$.

(ii) $X$ is not diffeomorphic to $Y$.

(iii) $X$ is hyperbolic.

(iv) $Y$ has sectional curvatures in $(-1-\epsilon,-1+\epsilon)$.

The technique mentioned above (proving Theorem 10) actually gives new cases also for the compact case, i.e., for Theorem 7.

Now, the negatively curved manifolds mentioned up to this point were homeomorphic (hence homotopy equivalent) to hyperbolic manifolds. We call these manifolds of hyperbolic homotopy type. In [7] Ardanza gave a version of Theorem 7 for manifolds that are not homotopy equivalent to a closed locally symmetric space; in particular, they do not have a hyperbolic homotopy type. His constructions use branched covers of hyperbolic manifolds. Recall that Gromov and Thurston [32] proved that large branched covers 
of hyperbolic manifolds do not have the homotopy type of a closed locally symmetric space. Here is the statement of Ardanza's result:

TheOREM 11. For all $n=4 k-1, k \geq 2$, there exist closed Riemannian $n$-dimensional manifolds $X$ and $Y$ with negative sectional curvature such that they do not have the homotopy type of a locally symmetric space and

(i) $X$ is homeomorphic to $Y$.

(ii) $X$ is not diffeomorphic to $Y$.

Later Farrell and Ontaneda [28] showed that most of the results mentioned in items 1-5 above are also true for examples of non-hyperbolic homotopy type.

Up to now the hyperbolic manifolds considered were real hyperbolic manifolds. We now consider Rigidity Questions for complex, quaternionic and Cayley hyperbolic manifolds. These are Riemannian $n$-manifolds whose universal covers, with the pulled back metric, are isometric to complex hyperbolic space $\mathbb{C} \mathbf{H}^{m}(n=2 m)$, quaternionic hyperbolic space $\mathbb{H} \mathbf{H}^{m}(n=4 m)$, or Cayley hyperbolic plane $\mathbb{O} \mathbf{H}^{2}(n=16)$, respectively. Recall that these manifolds have sectional curvatures in the interval $[-4,-1]$ and they also are rigid. In fact they satisfy the following stronger Rigidity results, called superrigidity in the quaternionic and Cayley cases. Assume that $X^{n}$ and $Y^{n}$ are homeomorphic closed Riemannian manifolds. Then:

(a) If $X$ is complex, quaternionic $(n=4 m, m \geq 2)$ or Cayley hyperbolic $(n=16)$ and $Y$ has sectional curvatures in $[-4,-1]$ then $X$ and $Y$ are isometric. This follows from results proved independently by Hernández [37] and Yau and Zheng [63].

(b) If $X$ is quaternionic $(n=4 m, m \geq 2)$ or Cayley hyperbolic $(n=16)$ and $Y$ has nonpositive curvature operator then $X$ and $Y$ are isometric (up to scaling). This follows from results proved Corlette [13].

(c) If $X$ is quaternionic $(n=4 m, m \geq 2)$ or Cayley hyperbolic $(n=16)$ and the complexified sectional curvatures of $Y$ are nonpositive then $X$ and $Y$ are isometric (up to scaling). This follows from results proved by Mok, Siu and Yeung [45].

REMARK. The conditions in items (a) or (b) for $Y$ imply the condition in (c) for $Y$.

But for the complex case (see item (a)) Farrell and Jones [21] proved that this Rigidity can not be strengthened to requiring that the curvatures lie in the interval $[-4-\epsilon,-1+\epsilon]$, for some $\epsilon>0$ :

TheOREM 12. For every integer $m$ of the form $4 k+1, k>1$ and $\epsilon>0$ there are closed Riemannian manifolds $X^{2 m}$ and $Y^{2 m}$ such that

(1) $X$ is homeomorphic but not diffeomorphic to $Y$.

(2) $X$ is complex hyperbolic.

(3) $Y$ has sectional curvatures in $[-4-\epsilon,-1+\epsilon]$. 
Also, Aravinda and Farrell proved similar results (see item (a) above) for the quaternionic [5] and Cayley [4] cases. Here is the statement for the quaternionic version of Theorem 12:

THEOREM 13. For $n=8,16$ any closed quaternionic hyperbolic manifold $X^{n}$ has a finite sheeted cover $Z$ such that if $\Sigma^{n}$ is an exotic $n$-sphere then

(1) $Z$ is not diffeomorphic to $Z \# \Sigma$.

(2) $Z \# \Sigma$ admits a Riemannian metric with negative sectional curvatures.

\section{REMARKS.}

1. In the Theorem above the conclusion remains true if we replace $Z$ by any finite sheeted cover of it.

2. The Theorem above holds also for $n=20$ with the extra condition: $6 \Sigma \neq 0$ in $\Theta_{20}$. The abelian groups $\Theta_{8}, \Theta_{16}$ and $\Theta_{20}$ have orders 2, 2,24 , respectively.

Here is the statement for the Cayley version of Theorem 12:

Theorem 14. Given $\epsilon>0$, any closed hyperbolic Cayley manifold $X^{16}$, and the unique exotic 16- sphere $\Sigma^{16}$ there is a finite sheeted cover $Z$ of $X$ such that

(1) $Z$ is not diffeomorphic to $Z \# \Sigma$.

(2) $Z \# \Sigma$ admits a Riemannian metric with sectional curvatures in $[-4-\epsilon,-1+\epsilon]$.

(3) Conclusions 1 and 2 remain true if $Z$ is replaced by any finite sheeted cover of it.

Finally Theorem 13 and 14 together with Corlette's superrigidity result (see item (b) above) were used by Aravinda and Farrell [6] to answer positively the following question posed by Petersen in his text book [51, pp. 239-240]:

- Question: Are there any closed smooth manifolds which support a negatively curved Riemannian metric but do not support a Riemannian metric with nonpositive curvature operator?

In fact the manifolds $Z \# \Sigma$ of theorems 13 and 14 provide such examples.

\section{Classification}

The idea of studying the space of all Riemannian metrics that satisfy some property is a very natural one and in this section we consider this, i.e., the Classification Question (1.6) mentioned in the Introduction, for the property negative (sectional) curvature. That is, we want to study the space of all negatively curved Riemannian metrics on a manifold. Let us introduce some notation. 
Let $X$ be a closed smooth manifold. We will denote by $\mathcal{M E} \mathcal{T}(X)$ the space of all Riemannian metrics on $X$ and we will consider $\mathcal{M E \mathcal { T }}(X)$ with the smooth topology. Note that the space $\mathcal{M E \mathcal { T }}(X)$ is contractible. A subspace of metrics whose sectional curvatures lie in some interval (closed, open, semi-open) will be denoted by placing a superscript on $\mathcal{M E \mathcal { T }}(X)$. For example, $\mathcal{M E} \mathcal{T}^{\sec <\epsilon}(X)$ denotes the subspace of $\mathcal{M E} \mathcal{T}(X)$ of all Riemannian metrics on $X$ that have all sectional curvatures less that $\epsilon$. Thus saying, for instance, that $X$ admits a negatively curved metric is equivalent to saying that $\mathcal{M E} \mathcal{T}^{\text {sec }}<0(X) \neq \emptyset$. Or, saying that all sectional curvatures of a Riemannian metric $g$ lie in the interval $[a, b]$ is equivalent to saying that $g \in \mathcal{M E} \mathcal{T}^{a \leq s e c} \leq b(X)$. Note that $\mathcal{M E}^{\text {sec }}=-1(X)$ is the space of hyperbolic metrics $\mathcal{H} y p(X)$ on $X$.

From now on we will assume that $\mathcal{M E T}^{\sec <0}(X) \neq \emptyset$. As mentioned above the idea of studying $\mathcal{M E \mathcal { T }}(X)^{s e c<0}$ is very natural. For example an open problem posed by K. Burns and A. Katok ([12], Question 7.1) is the following:

- Question: is the space $\mathcal{M E} \mathcal{T}^{\sec <0}(X)$ path connected?

Analogously, one can ask:

- Question: is (each component of) the space $\mathcal{M E \mathcal { T }}^{\text {sec }}<0(X)$ contractible?

- Question: is $\pi_{k}\left(\mathcal{M E} \mathcal{T}^{\sec <0}(X)\right)$ trivial?

In dimension two, Hamilton's Ricci flow [35] shows that $\mathcal{H} y p\left(X^{2}\right)$ is a deformation retract of $\mathcal{M E} \mathcal{T}^{\text {sec }}<0\left(X^{2}\right)$. But $\mathcal{H} y p\left(X^{2}\right)$ fibers over the Teichmüller space $\mathcal{T}\left(X^{2}\right) \cong \mathbb{R}^{6 \mu-6}$ ( $\mu$ is the genus of $X^{2}$ ), with contractible fiber $\mathcal{D}_{0}=\mathbb{R}^{+} \times D I F F_{0}\left(X^{2}\right)[\mathbf{1 4}]$. (Here $D I F F_{0}\left(X^{2}\right)$ consists of all selfdiffeomorphisms of $X^{2}$ which are homotopic to $i d_{X^{2}}$.) Therefore $\mathcal{H} y p\left(X^{2}\right)$ and $\mathcal{M E} \mathcal{T}^{\sec <0}\left(X^{2}\right)$ are contractible.

It was shown by Farrell and Ontaneda $[\mathbf{3 0}]$ that, for $n \geq 10$, $\mathcal{M E} \mathcal{T}^{\text {sec }}<0 \quad\left(X^{n}\right)$ is never path-connected; in fact, it has infinitely many path-components. Moreover they showed that all the groups $\pi_{2 p-4}\left(\mathcal{M E} \mathcal{T}^{\sec <0}\left(X^{n}\right)\right)$ are non-trivial for every prime number $p>2$, and such that $p<\frac{n+5}{6}$. (In fact, these groups contain the infinite sum $\left(\mathbb{Z}_{p}\right)^{\infty}$ of $\mathbb{Z}_{p}=\mathbb{Z} / p \mathbb{Z}$ 's, and hence they are not finitely generated.) They also showed that $\pi_{1}\left(\mathcal{M E} \mathcal{T}^{\sec <0}\left(X^{n}\right)\right)$ is not finitely generated when $n \geq 14$. These results about $\pi_{k}$ are true for each path component of $\mathcal{M E} \mathcal{T}^{\text {sec }<0}\left(X^{n}\right)$. Before we state the Main Theorem in $[\mathbf{3 0}]$, we need some definitions.

Denote by $D I F F(X)$ the group of all smooth self-diffeomorphisms of $X$. We have that $D I F F(X)$ acts on $\mathcal{M E \mathcal { T }}(X)$ pulling-back metrics. Note that $\operatorname{DIFF}(X)$ leaves invariant all spaces $\mathcal{M} \mathcal{E} \mathcal{T}^{\text {sec } \in I}(X)$, for any $I \subset \mathbb{R}$. For any metric $g$ on $X$ we denote by $D I F F(X) g$ the orbit of $g$ by the action of $\operatorname{DIFF}(X)$. We have a map $\Lambda_{g}: D I F F(X) \rightarrow \mathcal{M E \mathcal { T }}(X)$, given by $\Lambda_{g}(\phi)=\phi_{*} g$. Then the image of $\Lambda_{g}$ is the orbit DIFF(X)g of $g$. And $\Lambda_{g}$ of course naturally factors through $\mathcal{M} \mathcal{E} \mathcal{T}^{\text {sec } \in I}(X)$, if $g \in \mathcal{M E} \mathcal{T}^{\text {sec } \in I}(X)$. Note 
that if $\operatorname{dim} X \geq 3$ and $g \in \mathcal{M E} \mathcal{T}^{\text {secc=-1}}(X)$, then the statement of Mostow's Rigidity Theorem is equivalent to saying that the map $\Lambda_{g}: D I F F(X) \rightarrow$ $\mathcal{M E} \mathcal{T}^{\text {secc }}=-1(X)=\mathcal{H} y p(X)$ is a surjection. Here is the statement of the main result of $[\mathbf{3 0}]$ :

Theorem 16. Let $M$ be a closed smooth $n$-manifold and let $g$ be a negatively curved Riemannian metric on $X$. Then we have that:

(i) the map $\pi_{0}\left(\Lambda_{g}\right): \pi_{0}(\operatorname{DIFF}(X)) \rightarrow \pi_{0}\left(\mathcal{M E} \mathcal{T}^{\text {sec }}<0(X)\right)$ is not constant, provided $n \geq 10$.

(ii) the homomorphism $\pi_{1}\left(\Lambda_{g}\right): \pi_{1}(D I F F(X)) \rightarrow \pi_{1}\left(\mathcal{M E}^{\sec <0}(X)\right)$ is non-zero, provided $n \geq 14$.

(iii) For $k=2 p-4, p$ prime integer and $1<k \leq \frac{n-8}{3}$, the homomorphism $\pi_{k}\left(\Lambda_{g}\right): \pi_{k}(\operatorname{DIFF}(X)) \rightarrow \pi_{k}\left(\mathcal{M E T}^{\text {sec }<0}(X)\right)$ is nonzero.

Addendum to TheOREM 16. We have that the image of $\pi_{0}\left(\Lambda_{g}\right)$ is infinite and in cases (ii), (iii) mentioned in the Main Theorem, the image of $\pi_{k}\left(\Lambda_{g}\right)$ is not finitely generated. In fact we have:

(i) For $n \geq 10, \pi_{0}(\operatorname{DIFF}(X))$ contains $\left(\mathbb{Z}_{2}\right)^{\infty}$, and $\left.\pi_{0}\left(\Lambda_{g}\right)\right|_{\left(\mathbb{Z}_{2}\right)^{\infty}}$ is one-to-one.

(ii) For $n \geq 14$, the image of $\pi_{1}\left(\Lambda_{g}\right)$ contains $\left(\mathbb{Z}_{2}\right)^{\infty}$.

(iii) For $k=2 p-4, p$ prime integer and $1<k \leq \frac{n-8}{3}$, the image of $\pi_{k}\left(\Lambda_{g}\right)$ contains $\left(\mathbb{Z}_{p}\right)^{\infty}$.

We give two immediate corollaries. Note that for $a<b<0$ the map $\Lambda_{g}$ factors through the inclusion map $\mathcal{M} \mathcal{E} \mathcal{T}^{a \leq s e c} \leq b(X) \hookrightarrow \mathcal{M E}^{\sec <0}(X)$ provided $g \in \mathcal{M E} \mathcal{T}^{a \leq s e c \leq b}(X)$. Therefore we have:

Corollary 1. Let $X$ be a closed smooth $n$-manifold, $n \geq 10$. Let $a<$ $b<0$ and assume that $\mathcal{M E T}^{a \leq s e c \leq b}(X)$ is not empty. Then the inclusion map $\mathcal{M E T}^{a \leq s e c \leq b}(X) \hookrightarrow \mathcal{M E T}^{\text {sec }<0}(X)$ is not null-homotopic.

And if $a=b=-1$ we have:

Corollary 2. Let $X$ be a closed hyperbolic $n$-manifold, $n \geq 10$. Then the inclusion map $\mathcal{H} y p(X) \hookrightarrow \mathcal{M E} \mathcal{T}^{\text {sec }<0}(X)$ is not null-homotopic.

In the two Corollaries above we also have statements analogous to the cases (i), (ii), (iii) mentioned in the Main Theorem.

In particular the analogous case (i) for Corollary 2 implies that for any closed hyperbolic manifold $\left(X^{n}, g\right), n \geq 10$, there is a hyperbolic metric $g^{\prime}$ on $X$ such that $g$ and $g^{\prime}$ cannot be joined by a path of negatively curved metrics. Likewise, taking $a=-1-\epsilon, \quad b=-1(0 \leq \epsilon)$ in Corollary 1 we have that the space $\mathcal{M E} \mathcal{T}^{-1-\epsilon \leq s e c} \leq-1\left(X^{n}\right)$ of $\epsilon$-pinched negatively curved Riemannian metrics on $X$ has infinitely many path components, provided it is not empty 
and $n \geq 10$. And the homotopy groups $\pi_{k}\left(\mathcal{M E} \mathcal{T}^{-1-\epsilon \leq s e c} \leq-1(X)\right)$, are nonzero for the cases (ii.), (iii.) mentioned in the Main Theorem. Moreover, these groups are not finitely generated.

\section{The Teichmüller space of negatively curved metrics.}

Recall that we are denoting the group of all smooth self-diffeomorphisms of $X$ by $D I F F(X)$. Let $\mathcal{D}(X)$ be the group $\mathbb{R}^{+} \times D I F F(X)$. The group $\mathcal{D}(X)$ acts on $\mathcal{M E \mathcal { T }}(X)$ by scaling and pulling-back metrics: $(\lambda, \phi) g=$ $\lambda\left(\phi^{-1}\right)^{*} g=\lambda \phi_{*} g$, for $g \in \mathcal{M E \mathcal { T }}(X)$ and $(\lambda, \phi) \in \mathcal{D}(X)$. The quotient space $\mathcal{M}(X)=\mathcal{M E} \mathcal{T}(X) / \mathcal{D}(X)$ is called the moduli space of metrics on $X$. It is sometimes said that a geometric property is a property that is invariant by isometries, that is, by the action of $\operatorname{DIFF}(X)$. Clearly, the study of the moduli space of metrics is of fundamental importance not just in geometry but in other areas of mathematics as well. (See for instance Besse $[\mathbf{9}]$ Ch. 4). Also, denote by $D I F F_{0}(X)$ the subgroup of $D I F F(X)$ of all smooth diffeomorphisms of $X$ which are homotopic to the identity $1_{X}$ and by $\mathcal{D}_{0}(X)$ the group $\mathbb{R}^{+} \times D I F F_{0}(X)$. In $[\mathbf{2 9}]$ the Teichmüller space of metrics on $X$ is defined as the quotient space $\mathcal{T}(X)=\mathcal{M} \mathcal{E} \mathcal{T}(X) / \mathcal{D}_{0}(X)$.

It is interesting to consider subspaces of the space of metrics, the moduli space of metrics or the Teichmüller space that have geometric meaning. Given $0 \leq \epsilon \leq \infty$ let $\mathcal{M E \mathcal { T }}^{\epsilon}(X)$ denote the space of all $\epsilon$-pinched negatively curved Riemannian metrics on $X$, that is, $g \in \mathcal{M E}^{\epsilon}(X)$ if and only if there is a positive real number $\lambda$ such that $\lambda g$ has all its sectional curvatures in the interval $[-(1+\epsilon),-1]$. Note that a 0 -pinched metric is a metric of constant negative sectional curvature and an $\infty$-pinched metric is just a negatively curved Riemannian metric. The quotient space $\mathcal{M}^{\epsilon}(X)=\mathcal{M E}^{\epsilon}(X) / \mathcal{D}(X)$ is called the moduli space of $\epsilon$-pinched negatively curved metrics on $X$. Also, $\mathcal{T}^{\epsilon}(X)=\mathcal{M E}^{\epsilon} \mathcal{T}^{\epsilon}(X) / \mathcal{D}_{0}(X)$ is called the Teichmüller space of $\epsilon$-pinched negatively curved metrics on $X$. In particular, $\mathcal{T}^{\infty}(X)$ is the Teichmüller space of all negatively curved metrics on $X$. Note that the inclusions $\mathcal{M E}^{\epsilon}(X) \hookrightarrow \mathcal{M E} \mathcal{T}(X)$ induce inclusions $\mathcal{T}^{\epsilon}(X) \hookrightarrow \mathcal{T}(X)$.

The main result of Farrell and Ontaneda [29] states that for a hyperbolic manifold the map $\mathcal{T}^{\epsilon}(X) \hookrightarrow \mathcal{T}(X)$ is not in general homotopic to a constant map, provided $\epsilon>0$. In particular $\mathcal{T}^{\epsilon}(X), 0<\epsilon \leq \infty$, is in general not contractible. Here is a more detailed statement of this result:

Theorem 17. For every integer $k_{0} \geq 1$ there is an integer $n_{0}=n_{0}\left(k_{0}\right)$ such that the following holds. Given $\epsilon>0$ and a closed real hyperbolic $n$-manifold $X$ with $n \geq n_{0}$, there is a finite sheeted cover $Z$ of $X$ such that, for every $1 \leq k \leq k_{0}$ with $n+k \equiv 2 \bmod 4$, the $\operatorname{map} \pi_{k}\left(\mathcal{T}^{\epsilon}(Z)\right) \rightarrow$ $\pi_{k}(\mathcal{T}(Z))$, induced by the inclusion $\mathcal{T}^{\epsilon}(Z) \hookrightarrow \mathcal{T}(Z)$, is non-zero. Consequently $\pi_{k}\left(\mathcal{T}^{\epsilon}(Z)\right) \neq 0$. In particular, $\mathcal{T}^{\delta}(Z)$ is not contractible, for every $\delta$ such that $\epsilon \leq \delta \leq \infty$ (provided $k_{0} \geq 4$ ). 
REMARK. The homotopy groups $\pi_{k}$ in Theorem 17 are based at the class of the hyperbolic metric on $Z$.

As a Corollary of (proof of the) Theorem 17 we get:

Corollary. Let $X$ be a closed real hyperbolic manifold of dimension $n$, $n \geq 10$. Assume that $\Theta_{n+1} \neq 0$. Then for every $\epsilon>0$ there is a finite sheeted cover $Z$ of $X$ such that $\pi_{1}\left(\mathcal{T}^{\epsilon}(Z)\right) \neq 0$. Therefore $\mathcal{T}^{\epsilon}(Z)$ is not contractible.

\section{References}

[1] S.I. Al'ber, Spaces of mappings into manifold of negative curvature, Dokl. Akad. Nauk USSR, 168 (1968), 13-16.

[2] D. Anderson and W.-C. Hsiang, The functors $K_{i}$ and pseudoisotopies of polyhedra, Ann. of Math. (2), 105 (1977), 201-223.

[3] C.S. Aravinda and F.T. Farrell, Rank 1 aspherical manifolds which do not support any nonpositively curved metric, Comm. Anal. Geom., 2 (1994), 65-78.

[4] C.S. Aravinda and F.T. Farrell, Exotic negatively curved structures on Cayley hyperbolic manifolds, J. Differential Geom., 63 (2003), 41-62.

[5] C.S. Aravinda and F.T. Farrell, Exotic structures and quaternionic hyperbolic manifolds, in 'Algebraic Groups and Arithmetic', 507-524, Tata Inst. Fund. Res., Mumbai, 2004.

[6] C.S. Aravinda and F.T. Farrell, Nonpositivity: curvature vs. curvature operator, Proc. AMS, 132 (2005), 191-192.

[7] S. Ardanza, Ph.D. Thesis, Binghamton University, 2000.

[8] Bieberbach, Über die Bewegungsgruppen der Euklidischen Räume II, die Gruppen mit einen Fundamentalbereich, Math. Ann., 72 (1912), 400-412.

[9] A.L. Besse, Einstein Manifolds, Ergebnisse Series, 10, Springer-Verlag, Berlin, 1987.

[10] G. Besson, G. Courtois, and S. Gallot, Minimal entropy and Mostow's rigidity Theorems, Ergodic Theory \& Dynam. Sys., 16 (1996), 623-649.

[11] W. Browder, On the action of $\Theta^{n}(\partial \pi)$, Differential and Combinatorial Topology, Princeton University Press, Princeton, NJ, 1965, 23-36.

[12] K. Burns and A. Katok, Manifolds with nonpositive curvature, Ergodic Theory \& Dynam. Sys., 5 (1985), 307-317.

[13] K. Corlette, Archimedean superrigidity and hyperbolic geometry, Ann. of Math., 135 (1992), 165-182.

[14] C.J. Earle and J. Eells, Deformations of Riemannian surfaces, LNM, 102, SpringerVerlag, Berlin, 1969, 122-149.

[15] J. Eells and J.H. Sampson, Harmonic mappings of Riemannian manifolds, Amer. J. Math., 86 (1964), 109-160.

[16] F.T. Farrell and L.E. Jones, Negatively curved manifolds with exotic smooth structures, J. Amer. Math. Soc., 2 (1989), 899-908.

[17] F.T. Farrell and L.E. Jones, Rigidity in geometry and topology, Proc. of the ICM, Vol. I, II (Kyoto, 1990), Math. Soc. Japan, Tokyo, 1991, 653-663.

[18] F.T. Farrell and L.E. Jones, Compact infrasolvmanifolds are smoothly rigid, in 'Geometry from the Pacific Rim', edited by Berrick, Loo and Wang, de Gruyter \& Co., Berlin, 1997, 85-97.

[19] F.T. Farrell and L.E. Jones, Exotic smoothings of hyperbolic manifolds which do not support pinched negative curvature, Proc. Amer. Math. Soc., 121 (1994), 627-630.

[20] F.T. Farrell and L.E. Jones, Nonuniform hyperbolic lattices and exotic smooth structures, J. Differential Geom., 38 (1993), 235-261. 
[21] F.T. Farrell and L.E. Jones, Complex hyperbolic manifolds and exotic smooth structures, Invent. Math., 117 (1994), 57-74.

[22] F.T. Farrell, L.E. Jones, and P. Ontaneda, Hyperbolic manifolds with negatively curved exotic triangulations in dimension larger than five, J. Differential Geom., 48 (1998), 319-322.

[23] F.T. Farrell, L.E. Jones, and P. Ontaneda, Examples of non-homeomorphic harmonic maps between negatively curved manifolds, Bull. London Math. Soc., 30 (1998), 295296.

[24] F.T. Farrell, P. Ontaneda, and M.S. Raghunathan, Non-univalent harmonic maps homotopic to diffeomorphisms, J. Differential Geom., 54 (2000), 227-253.

[25] F.T. Farrell and P. Ontaneda, Harmonic cellular maps which are not diffeomorphisms, Inv. Math., 158 (2004), 497-513.

[26] F.T. Farrell and P. Ontaneda, Exotic structures and the limitations of certain analytic methods in geometry, Asian Jour. Math., 8 (2004), 639-652.

[27] F.T. Farrell and P. Ontaneda, A caveat on the convergence of the Ricci flow for negatively curved manifolds, Asian Jour. Math., 9 (2005), 401-406.

[28] F.T. Farrell and P. Ontaneda, Branched cover of hyperbolic manifolds and harmonic maps, Comm. in Analysis and Geometry, 14(2) (2006), 249-268.

[29] F.T. Farrell and P. Ontaneda, The Teichmüller space of pinched negatively curved metrics on a hyperbolic manifold is not contractible. To appear in Annals of Mathematics, ArxivmathDG.0406132.

[30] F.T. Farrell and P. Ontaneda, On the topology of the space of negatively curved metrics, Submitted for publication, Arxiv mathDG. 0607367.

[31] D. Gromoll and J. Wolf, Some relations between the metric structure and algebraic structure of the fundamental group in manifolds of nonpositive curvature, Bull. AMS, 77(4) (1971), 545-552.

[32] M. Gromov and W. Thurston, Pinching constants for hyperbolic manifolds, Invent. Math., 89 (1987), 1-12.

[33] M. Gromov and R. Schoen, Harmonic maps into singular spaces and p-adic superrigidity of lattices in groups of rank one, Inst. Hautes Études Sci. Publ. Math., 76 (1992), 165-246.

[34] R. Hamilton, Three-manifolds with positive Ricci curvature, J. Differential Geom., 17 (1982), 255-306.

[35] R. Hamilton, The Ricci flow on surfaces, Contemporary Mathematics, 71 (1988), 237-261.

[36] P. Hartman, On homotopic harmonic maps, Canad. J. Math., 19 (1967), 673-687.

[37] L. Hernández, Kähler manifolds and 1/4-pinching, Duke Math. J., 62 (1991), 601-611.

[38] G. Huisken, Ricci deformation of a metric on a Riemannian manifold, J. Differential Geom., 21 (1985), 47-62.

[39] J. Jost and S.-T. Yau, Harmonic maps and superrigidity, Proc. Sympos. Pure Math., 54, Amer. Math. Soc., Providence, RI, 1993, 245-280.

[40] H.B. Lawson and S.-T. Yau, Compact manifolds of nonpositive curvature, J. Differential Geom., 7 (1972), 211-228.

[41] K.-B. Lee and F. Raymond, Rigidity of almost crystallographic groups, Contemp. Math., 44, Amer. Math. Soc., Providence, RI, 1985, 73-78.

[42] C. Margerin, Pointwise pinched manifolds are space forms, Proc. Sympos. Pure Math., 44, Amer. Math. Soc., Providence, RI, 1986, 307-328.

[43] J.J. Millson, On the first Betti number of a constant negatively curved manifold, Ann. of Math., 104 (1976), 235-247.

[44] J.J. Millson and M.S. Raghunathan, Geometric construction of cohomology for arithmetic groups I, Proc. Indian Acad. Sci., 90(2) (1981), 103-123.

[45] N. Mok, Y.-T. Siu and S.-K. Yeung, Geometric superrigidity, Invent. Math., 113 (1993), 57-83. 
[46] G.D. Mostow, Quasi-conformal mappings in n-space and the rigidity of hyperbolic space forms, Inst. Hautes Études Sci. Publ. Math., 34 (1967), 53-104.

[47] S. Nishikawa, Deformation of Riemannian metrics and manifolds with bounded curvature ratios, Proc. Sympos. Pure Math., 44, Amer. Math. Soc., Providence, RI, 1986, 343-352.

[48] P. Ontaneda, Hyperbolic manifolds with negatively curved exotic triangulations in dimension six, J. Differential Geom., 40 (1994), 7-22.

[49] P. Ontaneda, The double of a hyperbolic manifold and exotic nonpositively curved structures, Trans. AMS, 40 (2000), 7-22.

[50] P. Ontaneda, A space with two nonpositively curved structures, Topology, 40 (2002), 7-22.

[51] P. Petersen, Riemannian Geometry, Graduate texts in Math., 171, Springer-Verlag, NY, 1998.

[52] J. Sampson, Some properties and applications of harmonic mappings, Ann. Scient. Ec. Norm. Sup., 11 (1978), 211-228.

[53] J. Sampson, Applications of harmonic maps to Kähler geometry, Cont. Math., 49 (1986), 125-133.

[54] Y.-T. Siu, The complex-analyticity of harmonic maps and the strong rigidity of compact Kähler manifolds, Ann. of Math., 112 (1980), 73-111.

[55] M. Scharlemann and L. Siebenmann, The Hauptvermutung for smooth singular homeomorphisms, in 'Manifolds' (Tokyo, 1973), Akio Hattori ed., Univ. of Tokyo Press, 85-91.

[56] M. Scharlemann, Smooth CE maps and smooth homeomorphisms, in 'Algebraic and Geometric Topology', A. Dold and B. Eckmann eds., Lecture Notes in Mathematics, 664, 234-240.

[57] R. Schoen and S.-T. Yau, On univalent harmonic maps between surfaces, Inv. Math., 44 (1978), 265-278.

[58] V. Schroeder, A cusp closing Theorem, Proc. AMS, 106 (1989), 797-802.

[59] C.W. Stark, Surgery theory and infinite fundamental groups, Ann. of Math. Studies, 145(1), 239-252.

[60] B. Wilking, Rigidity of group actions on solvable Lie groups, Math. Ann., 317 (2000), 195-237.

[61] S.-T. Yau, Seminar on differential geometry, Ann.of Math. Stud., 102, Princeton Univ. Press, Princeton, NJ, 1982.

[62] S.-T. Yau, On the fundamental group of compact manifolds of nonpositve curvature, Ann.of Math., 93 (1971), 579-585.

[63] S.-T. Yau and F. Zheng, Negatively 1/4-pinched Riemannian metric on a compact Kähler manifold, Invent. Math., 103 (1991), 527-535.

[64] R. Ye, Ricci flow, Einstein metrics and space forms, Transactions of the AMS, 338 (1993), 871-896.

SUNY, Binghamton, NY 13902, USA

E-mail address: farrell@math.binghamton.edu

SUNY, STony Brook, NY 11794, USA

E-mail address: lejones@math.sunysb.edu

SUNY, Binghamton, NY 13902, USA

E-mail address: pedro@math.binghamton.edu 
